\title{
Influences of Cloud Computing on E-Commerce Businesses and Industry
}

\author{
Danping Wang \\ Shanghai University of Finance and Economics, Shanghai, China. \\ Email: Nancyw1028@hotmail.com \\ Received April $2^{\text {nd }}, 2013$; revised May $3^{\text {rd }}, 2013$; accepted May $10^{\text {th }}, 2013$ \\ Copyright (C 2013 Danping Wang. This is an open access article distributed under the Creative Commons Attribution License, which \\ permits unrestricted use, distribution, and reproduction in any medium, provided the original work is properly cited.
}

\begin{abstract}
Cloud computing has been one of the most popular topics on information technology, since it was proposed by Google in 2007. In the past years, cloud computing has become from a theoretical concept into the real applications in different industries such as telecommunication and healthcare. Inevitably, its influences on different industries have also attracted much attention. Based on the literature review, this paper discussed how the traditional E-commerce businesses and industry were influenced by cloud computing in technical architecture, service modes and the industrial chain. Besides, it analyzed the driving-forces which led to the changes of E-commerce in cloud era. Finally, it concluded that only when the E-commerce enterprises involved cloud computing in the business strategy and established the core competencies, could they realize the sustainable development in the cloud era.
\end{abstract}

Keywords: Cloud Computing; E-Commerce; Business Model; Industry Chain

\section{Introduction}

Since it was proposed by Google in 2007, cloud computing has attracted a lot of attention. Cloud computing has developed quickly from a theoretical concept to the real applications in the past few years. More and more businesses and research agencies are striving to release cloud computing strategies and business models, perfect cloud computing technology, and propose the related applications of cloud computing. It enables the dynamic computing capacity, storage capacity, network exchanging capability and information service capability.

Cloud computing serves the users as "pay-as-service", which supplies and delivers the end users with IT services based on their demand. It departs the IT service processes and transfers them to the cloud platform, which leads to the new service modes such as IaaS (Infrastructure as a Service), PaaS (Platform as a Service) and SaaS (Software as a Service). As a new information means and mode, cloud computing is being applied to many industries creatively. E-commerce is a typical industry which is being influenced inevitably by the features of cloud computing. This paper discusses the impacts of cloud computing on the traditional E-commerce respectively from the perspective of technology, service and industry chain and presents the necessary suggestions on the de- velopment of E-commerce businesses in the cloud era.

\section{Literature Review}

Since the paper concentrates on how cloud computing influences E-commerce, the solid background of the impacts of cloud computing has to be established.

Since its emergence, the influences of cloud computing has been discussed by different literatures. Li J. and Liu J. [1] analyzes the factors such as the lack of teaching resources which are preventing the educational informationization in rural China and conclude that cloud computing can help to solve the problems. This paper finds the possible benefits to educational informationization brought by cloud computing. It is meaningful for the applications of cloud computing. However, all these possible benefits of cloud computing are only discussed rather than examined by the empirical study. Kashefi, F., et al. [2] consider the significant influences of cloud computing on the processes and present a new methodology of cloud computing. The paper describes the positive impacts of cloud computing based on the cases of the biggest companies and organizations such as Google, which makes the points more persuasive. Nevertheless, the authors emphasize the technical impacts of cloud computing rather than analyze its business impacts. 
Shuai Q. [3] analyzes the troubles faced by Chinese m-learning including limited resources of the mobile devices, storage capacity of massive didactical materials and expensive investment of hardware and software. The author points out that cloud computing, due to its reliable, customized and QoS guaranteed dynamic computing environments for end-users, has the capacity of solving the problems with m-learning. Finally, the author concludes that cloud computing would play a significant role in the improvement of m-learning. The paper predicts the application of cloud computing in m-learning. But it also lacks the empirical analysis. Lai, S. [4] analyzes the impact of cloud computing on the traditional software project and figures out that the traditional software project should migrate cloud computing. Especially, it proposes the migration strategies, security strategies and the corresponding migration tools. The contribution of the paper lies in that it not only describes the positive influences of cloud computing on the traditional software project but also offers the specific strategies by which cloud computing can be appropriately applied to the software project development. Zhang, H. [5] figures out that along with the rapid development of the economic environment, the virtual operation performance management is becoming more important. The core enterprises of the virtual organization need methods to achieve implementation of regulatory functions to the overall performance management objectives. Cloud computing technology will become the best choice of the virtual operation process due to its features such as reliability and security. The author also concludes that cloud computing would have the multiple effects on different levels and different aspects of performance management in virtual operation. There are two drawbacks of the paper: firstly, it does not analyze how cloud computing benefits the virtual operation performance management; secondly, it does not illustrate the specific examples to prove the points.

By virtue of the literature review, we can find that there are three problems with the existed researches: firstly, the existed literature usually focuses on one or two aspects of cloud computing's impacts on a specified field. There is no paper that offers the comprehensive analysis of the influences of cloud computing; Secondly, few literature mentions the influences of cloud computing on E-commerce. Actually, the rapid development of E-commerce requires the involvement of cloud computing into its technical architecture, business mode and service mode. As a result, the development of e-commerce businesses and industry will be significantly influenced. Finally, few literature supplies the case analysis to make the points more persuasive. By virtue of describing the changes of E-commerce in the cloud era, the paper analyzes the impacts of cloud computing on E-commerce enterprises and industry chain in detail.

\section{Influences on E-Commerce Technical Architecture}

E-commerce is the exchange of products and services via Internet. From the perspective of system, it is composed of two layers: one layer is the technical architecture made up of hardware and software; the other layer is the business transactions based on the technical architecture. According to Laudon [6], the technical architecture is the base of E-commerce. And only on the base of the technical architecture, can the E-commerce business modes and marketing strategies be realized. In addition, the security and stability of technical architecture are the premise of online products and services exchange. Cloud computing, a new computing mode, is going to make a significant impact on E-commerce technical architecture.

Actually, cloud computing is not a brand new computing mode. It is the evolution and extension of the traditional distributed computing and grid computing [7-16]. For instance, Weiss [7] defines cloud computing as the natural evolution and integration of effective computing, distributed and grid computing. Mell and Grance [17] defines cloud computing as a model which is able to visit the configurable resources such as network, server, memory, applications and services based on the demand. Therefore, the influences of cloud computing on Ecommerce technical architecture are demonstrated by the building, implementing and maintaining of the technical layer.

Firstly, cloud computing enables E-commerce enterprises to rent rather than purchase hardware and software, which helps them to decrease the cost of system building. Traditionally, E-commerce enterprises have to purchase all the needed hardware and software, which accounts for a high percentage of E-commerce operation expenses, especially for the medium and small businesses. By virtue of the cloud computing platform, an E-commerce enterprise can choose and rent the IT products and services based on its demand so as to establish the technical architecture. Especially, the charging mode of "pay-asservice" is very flexible, which helps an E-commerce company to pay for the resources based on the demand. EC2 (Elastic Compute Cloud), a cloud system offered by Amazon, which allows the users to rent the applications in the cloud is a good example. Many businesses have benefited from EC2. For example, The New York Times, a very popular newspaper chose EC2 when it planned to transform all the TIF data into PDF data. As a result, it only cost 3000 US dollar to achieve the goals. The powerful computing ability of cloud computing enables the dramatically reduced data transformation cost. Another similar example is from Eli Lilly \& Co., a big medical company. Using EC2, Eli Lilly \& Co., decreased the cost of data analysis to 89 US dollar, which usually spent 
more than one million dollars before.

Secondly, cloud computing solved the problem of resources utilization efficiency. For an E-commerce company, it is necessary to invest on the software and hardware to maintain the operation. With the company's growth, the investment will be increased. However, the utilization efficiency of the invested infrastructure is low due to the sales season and the change consumers' behavior. According to Robert L. [18], the average utilization efficiency is no more than $10 \%$, which causes the IT resources waste. Cloud computing enables the businesses to integrate the idle IT resources (e.g. server) on the farend platform and rent them to the customers. This mode on the one hand reduces the operation cost of an E-commerce company and prioritizes resources allocation on the other hand.

Though cloud computing benefits the building and implementing of E-commerce technical architecture, the problems of system security and stability with it will be a problem non-neglectable. When all the IT resources such as hardware, software, data and network applications are stored on the cloud platform as services, users will unavoidably concern about the security and stability of the platform. Once the cloud platform is attacked, the important data of E-commerce transactions will be lost. Besides, customers' privacy may become an obstacle for the cloud applications of E-commerce.

\section{Influences on E-Commerce Backend Service Mode}

The new service mode offered by cloud computing differentiates it from the traditional IT services. Firstly, all the IT resources such as hardware, software, data and infrastructure are offered to the E-commerce enterprises as service by virtue of the cloud platform $[18,19]$. Secondly, just like the utility services (e.g. electricity), an E-commerce company is allowed to access to the IT resources on the cloud platform and pay for them as services [8]. It does not require the high expenses on devices purchase and each firm is able to choose the appropriate IT resources through renting. In another word, the emergence of cloud computing brings the new service philosophy and mode which enables the lower cost and changes the traditional IT licensing mode.

A significant contribution of cloud computing to Ecommerce lies in that it migrates outsourcing into Ecommerce. As the outsourcing service provider, a cloud computing business establishes the standardized and uniform service platform which integrates the infrastructure, application software and developing environment and customize them based on customers' demand. As the customer, an E-commerce enterprise searches the needed services and pays for it based on the demand. Essentially, it is a kind of outsourcing, that is, the E-commerce com- pany delivers the backend processes that it had to complete before to the cloud computing provider through contracting. The backend service mode based on cloud computing changes the close-end service that the service provider has to send the IT personnel to conduct local technical support and is called as "outsourcing 2.0" [20].

The primary objective of outsourcing is to reduce cost, improve efficiency as well as service quality and improve the core competencies of an organization. The evolution of E-commerce indicates that the core competency of an E-commerce firm is not the pure technology any longer but the business or service mode. Cloud computing sets the E-commerce enterprises free from the complicated technical architecture planning, designing and maintaining and enables them to focus on the core businesses. Virtual business is the typical example of the new outsourcing based on cloud computing. It refers to that an E-commerce firm fulfills most of its functionalities through "cloud-outsourcing" [21]. The advantages of virtual business lie in 1) The charging mode of "payas-service" enables the cost saving; 2) The expenses of devices upgrading can be decreased; 3) The "cloud-outsourcing" mode enables the higher visiting of E-commerce website.

\section{Influences on E-Commerce Business Strategies}

Since the emergence of cloud computing, a lot of Ecommerce firms begin to expand their business to cloud computing. Some famous E-commerce businesses such as Amazon, Google and Alibaba have involved cloud computing in their long-term strategies.

Several driving forces lead to the migration of cloud computing into E-commerce strategies: 1) Demand. With the rapid development of information technology, E-commerce services are improving - the services with higher efficiency, lower cost, more flexibility and diversity are needed. For instance, Alibaba, the biggest B2B E-commerce enterprise offers the online loan services by virtue of cloud computing. Alibaba loans the small and medium businesses its own idle capital from B2B transactions. When evaluating a customer's creditability, Alibaba implements the quick data analysis with cloud computing, which ensure the efficiency and effectiveness of creditability evaluation; 2) Efficiency. The efficiency advantages of cloud computing lies in two aspects: on the one hand, the huge data storage is becoming a problem with the growth of E-commerce firms. Establishing the data center is unaffordable for medium and small E-commerce enterprises. Cloud computing enables the outsourcing of the backend infrastructure building and application software services. In addition, the "pay-as-service" charging mode greatly reduces the operation cost 
and the environment pollution. On the other hand, the excellent ability of data integration and processing remarkably increase the efficiency of online services; 3 ) Policy. Government's policy is another driving force which spurs the E-commerce enterprises to involve cloud computing into their business strategies. In 2009, Obama - president of U.S.A announced a long-term cloud computing plan so as to reduce government's expenses by virtualization technology. In the same year, Apps.gov-a cloud services website was established. This online store enables all the federal institutions to browse and purchase IT services based on cloud computing. These policies and measures indicate that cloud services will help E-commerce enterprises to gain more opportunities and resources; 4) Quality. Customers' higher requirement on the quality of E-commerce products and services is another driving force which makes the E-commerce enterprises implement their cloud strategies. For example, Baidu, the largest Chinese searching engine, in 2011, announced opening its cloud platform which supports 6 billion searching from the 138 countries. Cloud computing enables Baidu the functionalities through the ability of huge data storing and processing, real-time computing and high quality service architecture. As a result, Baidu stands out among the information searching platforms due to its ability of quick response and high reliability of 99.999\%.

\section{Influences on E-Commerce Industry Chain Structure}

Cloud computing may influence the traditional E-commerce industry chain and lead to the chain restructuringTraditionally, the E-commerce industry chain is composed of the hardware supplier, software developer,
Internet service provider, system integrating provider, service supplier, E-commerce enterprise and customer (Figure 1). Each member of the industry chain fulfills its own functionalities. The hardware supplier, software developer, Internet service provider, system integration provider, service supplier exist as the backend of the E-commerce enterprise and offers it the technical support.

When cloud computing is migrated into E-commerce industry, one cloud service provider can supply almost all the necessary products and services to an E-commerce website. As a result, the structure of E-commerce industry chain will be changed (Figure 2).

On the one hand, an E-commerce enterprise doesn't have to purchase IT resources. Instead, it just rents the cloud services needed. Thus, the profiting space of the traditional IT firms (e.g. IT service provider) in the chain will become smaller inevitably. They may cooperate with a cloud service provider and become its "backend" which offers the necessary infrastructure services for the cloud service provider. The E-commerce enterprise will be directly served by the cloud service provider rather than the IT firms group. Fatherly, with the popularity of cloud computing, the end users who buy the hardware or software products from the traditional E-commerce websites will probably transfer to the cloud computing platform which offers all the IT resources as services. That means the IT resources market of the traditional E-commerce website will be partly shared by cloud computing. The E-commerce enterprises lacking the core competencies will have to exit from the market. Eventually, the E-commerce industry chain will be restructured.

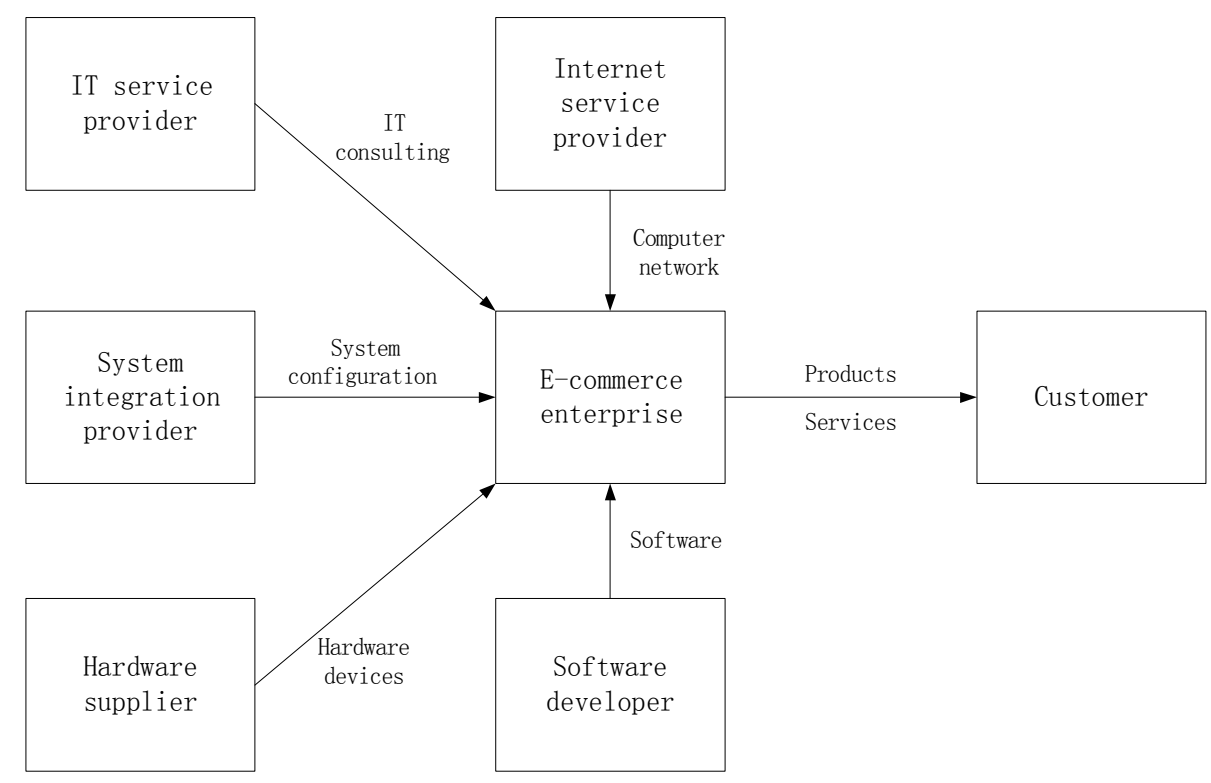

Figure 1. Traditional E-commerce industry chain. 


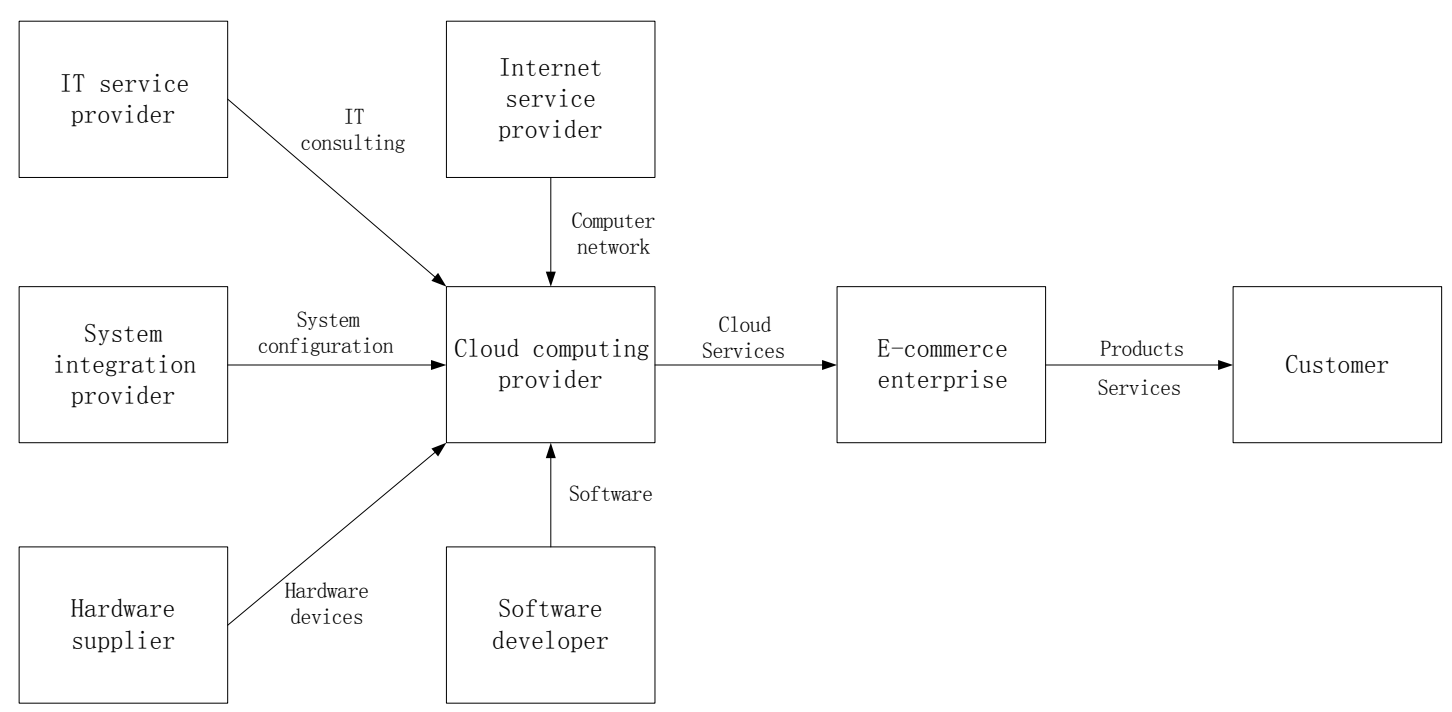

Figure 2. E-commerce industry chain based on cloud computing.

\section{Suggestions on E-Commerce Enterprises' Development in the Cloud Era}

How does an E-commerce enterprise cope with the challenges brought by cloud computing since it greatly influences E-commerce? And how does an E-commerce enterprise seize the opportunities offered by cloud computing?

Firstly, an E-commerce enterprise should concentrate on the core competencies. Since E-commerce is a kind of new business mode by virtue of computer network, an E-commerce enterprise should emphasize the operation and management. Actually, cloud computing will threaten the traditional IT enterprises including E-commerce firms. For example, customers are able to utilize the software by the "pay-as-service" charging mode rather than buy it from the traditional E-commerce website. The market share of the E-commerce enterprises which focus on the online software sales will decrease greatly. As what Nicholas Carr [22] said, the development of cloud computing will cause the information technology to become the inexpensive public resource which is accessible to all the people. As a result, information technology will not be the core competency of an E-commerce enterprise. Therefore, an E-commerce enterprise should emphasize the business operation and management more.

Secondly, an E-commerce enterprise should focus on the innovation of business mode and operation mode since they are the critical factors for an enterprise's success. For example, Alibaba began to build its E-commerce cloud center since 2009. It plans to integrate the cloud center with the existed data center and establish the business cloud which can compete with the famous cloud services providers such as Google. In addition, Alibaba supplies the customized and personalized cloud services such as application engine, application framework and resources searching for the small and medium businesses. The business mode differentiates Alibaba from other Ecommerce competitors. By virtue of the business cloud offered by Alibaba, users don't have to concern about the complex IT infrastructure and only need to rent the cloud services based on the demand to achieve the goals.

Finally, an E-commerce enterprise should cooperate with cloud service provider so as to improve the operation capability. Since the cloud computing development is unaffordable for most of E-commerce enterprises, it requires the close cooperation with partners. The cooperation with cloud service provider enables the E-commerce enterprise especially the medium and small enterprises to find a new way.

\section{Conclusions}

The emergence of cloud computing is creating a new service ecosystem which will integrate all the E-commerce resources and facilitate the new service modes. For the E-commerce providers, cloud computing is creating the good opportunities while threatening their existence.

For the traditional E-commerce enterprises, an appropriate strategy of development in the cloud era is to embrace cloud computing rather than resist on it. Only when the E-commerce enterprises involve cloud computing in the business strategy and establish the core competencies, can they realize the sustainable development.

\section{REFERENCES}

[1] J. Li and J. Z. Liu, "Influence of Cloud Computing on Educational Informationization of China Rural Areas," The Proceedings of Information Science and Engineering 
Conference, Hangzhou, 4-6 December 2010, pp. 281-283.

[2] F. Kashefi, M. Majd, M. Darbandi, H. Purhosein, K. Alizadeh and O. Atae, "Perusal about Influences of Cloud Computing on the Processes of These Days and Presenting New Ideas about Its Security," The Proceedings of the 5th International Conference on Application of Information and Communication Technologies (AICT), Baku, 12-14 October 2011, pp. 1-4.

[3] S. Qin, "What Will Cloud Computing Provide for Chinese M-Learning?" The Proceedings of International Conference on E-Education, Entertainment and E-Management, Bali, 27-29 December 2011, pp. 171-174.

[4] S. L. Lai, "The Influences of Cloud Computing to the Traditional Software Project and Our Corresponding Strategies," The Proceedings of the 3rd International Conference on Intelligent System Design and Engineering Applications, Hong Kong, 16-18 January 2013, pp. 14611464.

[5] H. X. Zhang, "Research on the Influence of Cloud Computing on the Virtual Operation Performance Management," The proceedings of the 7th International Conference on Computer Science \& Education, Melbourne, 1417 July 2012, pp. 235-238.

[6] K. C. Laudon and C. G. Traver, "E-Commerce: Business, Technology, Society,” 2nd Edition, Addison Wesley Publish, Boston, 2001.

[7] A. Weiss, "Computing in the Cloud," ACM NetWorker Vol. 11, No. 4, 2007, pp. 16-25.

[8] L. Z. Wang and G. von Laszewski, "Scientific Cloud Computing: Early Definition and Experience,” Proceedings of High Performance Computing and Communications, Dalian, 25-27 September 2008, pp. 825-830.

[9] B. Hayes, "Cloud Computing," Communications of the ACM, Vol. 51, No. 7, 2008, pp. 9-11. doi:10.1145/1364782.1364786

[10] J. Staten, et al., "Is Cloud Computing Ready for the Enterprise?” Forrester Research, 2008.

[11] F. M. Aymerich, G. Fenu1 and S. Surcis, "An Approach to a Cloud Computing Network," Proceedings of Applications of Digital Information and Web Technologies,
Ostrava, 4-6 August 2008, pp. 113-118.

[12] R. Buyya1, C. S. Yeo and S. Venugopal, "Market-Oriented Cloud Computing: Vision, Hype, and Reality for Delivering IT Services as Computing Utilities,” 10th IEEE International Conference on High Performance Computing and Communications, Dalian, 25-27 September 2008, pp. 5-13.

[13] B. P. Rimal and E. Choi, “A Conceptual Approach for Taxonomical Spectrum of Cloud Computing," The Proceedings of the 4th International Conference on Ubiquitous Information Technologies \& Applications, Fukuoka, 20-22 December 2009, pp. 1-6.

[14] B. P. Rimal, E. Choi and I. Lumb, "A taxonomy and Survey of Cloud Computing Systems," The Proceedings of 5th International Joint Conference on INC, IMS and IDC, Seoul, 25-27 August 2009, pp. 44-51.

[15] P. W. Hu and F. X. Hu, “An Optimized Strategy for Cloud Computing Architecture," The Proceedings of Conference on Computer Science and Information Technology (ICCSIT), Chengdu, 9-11 July 2010, pp. 374-378.

[16] J. Foster, Y. Zhao, I. Raicu and S. Y. Lu, "Cloud Computing and Grid Computing 360-Degree Compared,” Proceedings of Grid Computing Environments Workshop, Austin, 12-16 November 2008, pp. 1-10.

[17] P. Mell and T. Grance, "The NIST Definition of Cloud Computing,” 2010. http://www.blogjava.net/zamber/archive

[18] R. L. Grossman, "The Case for Cloud Computing, IT Professional, Vol. 11, No. 2, 2009, pp. 23-27.

[19] G. Boss, P. Malladi, D. Quan, L. Legregni and H. Hall, "Cloud Computing," IBM White Paper, 2007. http://download.boulder.ibm.com/ibmdl/pub/software/dw/ wes/hipods/Cloud_computing_wp_final_8Oct.pdf

[20] H. T. Qi, "Serving in the Cloud," Finance Outsourcing, 2010.

[21] H. R. Motahari-Nezhad, B. Stephenson and S. Singhal, "Outsourcing Business to Cloud Computing Services: Opportunities and Challenges,” HP Laboratories.

[22] N. Carr, “The Big Switch,” Zhongxin Publish, Beijing, 2008. 